
\title{
25 Research Soure \\ Beta-Lactam Dosing During Continuous Renal Replacement Therapy: A Survey of Practices in French Intensive Care Units
}

Elodie Matusik ( $\nabla$ elodie.matusik@gmail.com )

Centre Hospitalier de Valenciennes https://orcid.org/0000-0003-3046-145X

Justine Lemtiri

Hospital Centre Valenciennes: Centre Hospitalier de Valenciennes

Guillaume Wabont

Hospital Centre Valenciennes: Centre Hospitalier de Valenciennes

Fabien Lambiotte

Hospital Centre Valenciennes: Centre Hospitalier de Valenciennes

\section{Research}

Keywords: Beta lactams, pharmacokinetics, renal replacement therapy, critical illness, surveys and questionnaires

Posted Date: August 17th, 2021

DOl: https://doi.org/10.21203/rs.3.rs-800962/v1

License: (9) (1) This work is licensed under a Creative Commons Attribution 4.0 International License. Read Full License

Version of Record: A version of this preprint was published at BMC Nephrology on January 29th, 2022. See the published version at https://doi.org/10.1186/s12882-022-02678-x. 


\section{Abstract}

\section{Background}

Little information is available on current practice in beta-lactam dosing during continuous renal replacement therapy (CRRT). Optimized dosing is essential for improving outcomes, and there is no consensus on the appropriate dose regimens. The objective of the present study was to describe current practice for beta-lactam dosing during CRRT in intensive care units (ICUs).

\section{Methods}

We conducted a nationwide survey by e-mailing an online questionnaire to physicians working in ICUs in France. The questionnaire included three sections: demographic characteristics, CRRT practices, and beta-lactam dosing regimens during CRRT.

Results

157 intensivists completed the questionnaire. Continuous venovenous hemofiltration was the most frequently used CRRT technique, and citrate was the most regularly used anticoagulant. The median prescribed dose at baseline was $30 \mathrm{~mL} / \mathrm{kg} / \mathrm{h}$. The majority of prescribers (57\%) did not reduce betalactam dosing during CRRT. The tools were used to adapt dosing regimens during CRRT included guidelines, therapeutic drug monitoring (TDM), and data from the literature. When TDM was used, 100\% $\mathrm{T}>4$ time the MIC was the most common mentioned pharmacokinetic/pharmacodynamic target (53\%). Pharmacokinetic software tools were rarely used. Prolonged or continuous infusions were widely used during CRRT (88\%). Institutional guidelines on beta-lactam dosing during CRRT were rare. $41 \%$ of physicians sometimes consulted another specialist before adapting the dose of antibiotic during CRRT.

\section{Conclusions}

Our present results highlight the wide range of beta-lactam dosing practices adopted during CRRT. Personalized TDM and the implementation of Bayesian software appear to be essential for optimizing beta-lactam dosing regimens and improving patient outcomes.

\section{Background}

Beta-lactams are the most widely prescribed antibiotics in critically ill patients. Optimized dosing of betalactams is required to deal with pharmacokinetic changes and frequent underdosing - especially during the early phase of sepsis [1-3]. Renal failure may protect patients from insufficient antibiotic exposure by increasing the probability of beta-lactam target concentration attainment [2-5], and patients may benefit from high doses - particularly during the first 24 to 48 hours of antibiotic treatment $[3,6]$. Several experts have called for caution when using reduced dosing regimens in patients receiving continuous renal replacement therapy (CRRT) [7-8]. Seyler et al. demonstrated the inadequacy of the recommended betalactam dosing regimens during CRRT when bacteria with a high minimal inhibitory concentration (MIC) 
are involved [9]. Underdosing has prompted several experts to suggest the use of non-adjusted dosing within the first 24 to 48 hours [10-14], albeit with a potential risk of overdose [12-15]. Although betalactam dosing can be guided by clinical and pharmacokinetic data from the literature, the studies concern mainly intermittent hemodialysis and cannot be applied to CRRT, for which a variety of practices are used [15-16]. Li and Vaara highlighted the lack of key information required to correctly interpret studies and devise dose adjustments [18-19]. In 2019, these marked differences in CRRT practices and the subsequent influence on beta-lactam elimination prompted two French learned societies to recommend therapeutic drug monitoring (TDM) of beta-lactams in patients receiving CRRT [5]. In 2020, several international learned societies recommended TDM for routine use in critically ill patients [20]. However, TDM is not often available outside university hospitals. Given these uncertainties and our experience that neurotoxicity is more frequent in patients receiving CRRT, we decided to survey beta-lactam dosing practices during CRRT in France.

\section{Methods}

\section{Survey development}

Given the absence of data on the beta-lactam dosing prescribed during CRRT in France, we designed a survey to assess current practices. It was developed by a pharmacy resident with help from a critically ill clinical pharmacist and an intensivist, after a review of the literature. We performed an online, nationwide, cross-sectional survey between July and September 2019 by emailing a questionnaire to 1423 senior physicians working in intensive care units (ICUs) across France. The survey included 22 questions on the respondents' characteristics, CRRT practices, and beta-lactam dosing regimens during CRRT. The English version of the questionnaire and the results are given in Table 1. In order to determine which membrane material was used, brand names were cited in the questionnaire. Three clinical vignettes describing a critically ill patient weighing $70 \mathrm{~kg}$ and being treated for infectious pneumonia with piperacillintazobactam, cefotaxime or meropenem were used to prompt respondents to describe their beta-lactam dosing practices during CRRT (at $25 \mathrm{ml} / \mathrm{kg} / \mathrm{h}$ ). The questionnaire was made available on Google Forms $\circledast$ (Google, Inc., Mountain View, CA, USA). Data were extracted into an Excel® spreadsheet (Microsoft Corp, Redmond, WA, USA). Participation was anonymous. According to French legislation, approval by an investigational review board was not required for this survey. 


\section{Questions}

Responses are $\mathrm{n} / \mathrm{N}(\%)$ unless otherwise indicated

\section{Demographic characteristics}

What is your medical qualification?

Critical care medicine

77/157 (49)

Anesthesiology

61/157 (39)

Pulmonology

$5 / 157(3)$

Nephrology

$5 / 157(3)$

Emergency

$4 / 157$ (3)

Internal medicine

2/157 (1)

Cardiology

2/157 (1)

Infectious disease

$1 / 157(1)$

For how many years have you worked in an ICU (years, median [IQR])

$10[4-18]$

In which type of institution do you work?

University hospital

$78 / 157(50)$

Public-sector general hospital

$71 / 157(45)$

Private-sector for-profit or non-profit hospital

$8 / 157(5)$

\section{CRRT practices}

Which RRT modality do you most commonly use?

Continuous renal replacement therapy

$110 / 157(70)$

Intermittent renal replacement therapy

$47 / 157(30)$

Which CRRT techniques do you use?

Continuous venovenous hemofiltration

$114 / 157(71)$

Continuous venovenous hemodialysis

$89 / 157(57)$

Continuous venovenous hemodiafiltration

$87 / 157(55)$

Sustained Low-Efficiency Dialysis

$13 / 157(8)$

Abbreviations: CRRT: continuous renal replacement therapy, MIC: minimum inhibitory concentration, BID: twice a day, TID: three times a day, QID: four times a day 
Which is the most commonly used CRRT technique?

Continuous venovenous hemofiltration

Continuous venovenous hemodialysis

Continuous venovenous hemodiafiltration

Which is the most commonly used anticoagulant?

Citrate

Heparin

If you use continuous venovenous hemofiltration or hemodiafiltration, which hemofiltration mode do you prefer?

Postdilution mode

Predilution mode

Pre/postdilution mode

Which is the most commonly used CRRT dose, and how do you adjust it?

$20 \mathrm{~mL} / \mathrm{kg} / \mathrm{h}$

$25 \mathrm{~mL} / \mathrm{kg} / \mathrm{h}$

$30 \mathrm{~mL} / \mathrm{kg} / \mathrm{h}$

$35 \mathrm{~mL} / \mathrm{kg} / \mathrm{h}$

$2000 \mathrm{~mL} / \mathrm{h}$ (effluent flow not adjusted for body weight)

$2500 \mathrm{~mL} / \mathrm{h}$ (effluent flow not adjusted for body weight)

$3000 \mathrm{~mL} / \mathrm{h}$ (effluent flow not adjusted for body weight)

Dialysis dose adjusted for body weight upon CRRT initiation

Dialysis dose adjusted for body weight on admission

Dialysis dose adjusted for ideal total weight

Which type(s) of membrane do you use for CRRT?
$81 / 156(52)$

$54 / 156(35)$

$21 / 156(13)$

$100 / 157(64)$

$57 / 157(36)$

$22 / 142(16)$

$11 / 142(8)$

$106 / 142(77)$

9/157 (6)

$36 / 157(23)$

$49 / 157(31)$

$45 / 157(29)$

$12 / 157(8)$

$3 / 157(2)$

$3 / 157(2)$

29/75 (39)

$27 / 75(36)$

$19 / 75(25)$

Abbreviations: CRRT: continuous renal replacement therapy, MIC: minimum inhibitory concentration, BID: twice a day, TID: three times a day, QID: four times a day 
; Theradial® kits: Aquamax HF12, HF 19)

Polyarylethersulfone (Baxter® kits: Prismaflex HF1000, HF1400)

9/138 (7)

Acrylonitrile (Baxter ${ }^{\circledR}$ kits: Prismaflex M100, M150)

Acrylonitrile coated with polyethylenimine (Baxter® kits: Prismaflex ST100, ST150)

\section{Beta-lactam dosing regimens during CRRT}

Which beta-lactam dosing regimen do you prescribe for patients on CRRT?

Unadjusted dosing regimens

$88 / 157(56)$

Full dose for 24 hours and then a reduced-dosing regimen 26/157 (17)

Full dose for 48 hours and then a reduced-dosing regimen $14 / 157(9)$

A single loading dose before a reduced-dosing regimen 20/157 (13)

Reduced-dosing regimens all the time

$3 / 157(2)$

Reduced or full doses, depending on the drug compound

$6 / 157(4)$

Do you adjust the antibiotic dose based on the dialysis dose or effluent flow?

Yes

23/154 (15)

No

Do you use prolonged/continuous infusions for beta-lactams in patients on CRRT?

Yes

No

If yes, for which beta-lactam?

Piperacillin/tazobactam

Cefotaxime

Ceftazidime

Cefepime

$59 / 119(50)$

Abbreviations: CRRT: continuous renal replacement therapy, MIC: minimum inhibitory concentration, BID: twice a day, TID: three times a day, QID: four times a day 
If yes, which tools do you use?

Guide Prescription et Rein (French renal prescription handbook)

Therapeutic drug monitoring

Data from clinical studies in the literature

Dosing regimens of patients with renal failure applied to the estimated creatinine

$6 / 139(4)$ clearance rate of the patient on RRT

Pharmacokinetic software

Pharmacokinetic calculations by hand

Other tools

If beta-lactam therapeutic drug monitoring is used, which

pharmacokinetic/pharmacodynamic target do you use?

$40 / 50 / 70 \%$ T $>$ MIC

0/74 (0)

$100 \% \mathrm{~T}>\mathrm{MIC}$

12/74 (16)

$40 / 50 / 70 \%$ T > 4 MIC

4/74 (5)

$100 \% \mathrm{~T}>4 \mathrm{MIC}$

39/74 (53)

$40 / 50 / 70 \%$ T > 5 MIC

0/74 (0)

$100 \% \mathrm{~T}>5 \mathrm{MIC}$

7/74 (10)

$40 / 50 / 70 \%$ T > 8 MIC

0/74 (0)

$100 \% \mathrm{~T}>8 \mathrm{MIC}$

12/74 (16)

Do you sometimes call other specialists for advice on antibiotic dosing regimen adjustment for patients on CRRT?

No

Infectious disease specialist

Microbiologist

$16 / 157(10)$

Pharmacist/pharmacologist

$13 / 157(8)$

Abbreviations: CRRT: continuous renal replacement therapy, MIC: minimum inhibitory concentration, BID: twice a day, TID: three times a day, QID: four times a day 
For a $70 \mathrm{~kg}$ patient admitted with community-acquired infectious pneumonia and treated with your preferred CRRT technique at $25 \mathrm{ml} / \mathrm{kg} / \mathrm{hour}$, which maintenance dose do you prescribe for cefotaxime?

For a $70 \mathrm{~kg}$ patient admitted with hospital-acquired infectious pneumonia and treated with your preferred CRRT technique at $25 \mathrm{ml} / \mathrm{kg} / \mathrm{hour}$, which maintenance dose do you prescribe for piperacillin/tazobactam?

$\begin{array}{lc}4 / 0.5 \mathrm{~g} \text { QID } & 61 / 154(40) \\ 4 / 0.5 \mathrm{~g} \text { TID } & 69 / 154(45) \\ 4 / 0.5 \mathrm{~g} \mathrm{BID} & 15 / 154(10) \\ 3 / 0.375 \mathrm{~g} \text { QID } & 6 / 154(4) \\ \text { Other } & 2 / 154(1)\end{array}$

For a $70 \mathrm{~kg}$ patient admitted with hospital-acquired infectious pneumonia and treated with your preferred CRRT technique at $25 \mathrm{ml} / \mathrm{kg} / \mathrm{hour}$, which maintenance dose do you prescribe for meropenem?

$2 \mathrm{~g}$ TID

$2 \mathrm{~g} \mathrm{BID}$

$1 \mathrm{~g}$ TID

$1 \mathrm{~g} \mathrm{BID}$

Other
$48 / 156(31)$

9/156 (6)

$69 / 156(44)$

28/156 (18)

$2 / 156(1)$

Does your institution have procedures for adjusting antibiotic doses in patients on CRRT?

Yes

$33 / 157(21)$

Abbreviations: CRRT: continuous renal replacement therapy, MIC: minimum inhibitory concentration, BID: twice a day, TID: three times a day, QID: four times a day 


\begin{tabular}{|l|l|}
\hline Questions & $\begin{array}{l}\text { Responses are } \\
\text { n/N (\%) unless } \\
\text { otherwise } \\
\text { indicated }\end{array}$ \\
\hline No & \begin{tabular}{c}
$124 / 157(79)$ \\
\hline $\begin{array}{l}\text { Do you feel that you observe more beta-lactam-induced neurotoxicity in patients } \\
\text { treated with renal replacement than in other patients? }\end{array}$ \\
\hline Fully agree
\end{tabular} \\
\hline Tend to agree & $5 / 157(3)$ \\
\hline Tend to disagree & $48 / 157(31)$ \\
\hline Strongly disagree & $80 / 157(51)$ \\
\hline $\begin{array}{l}\text { Abbreviations: CRRT: continuous renal replacement therapy, MIC: minimum inhibitory concentration, } \\
\text { BID: twice a day, TID: three times a day, QID: four times a day }\end{array}$ & $24 / 157(15)$ \\
\hline
\end{tabular}

\section{Statistical analysis}

The results were presented as the frequency (percentage) for qualitative variables and the median [interquartile range (IQR)] for quantitative variables. For statistical comparisons of different groups, we applied Pearson's chi-square test with Yates' correction. All tests were two-sided, and the threshold for statistical significance was set to $p<0.05$. Statistical tests were performed using $S A S \circledast$ software (version 3.8, SAS Institute, Cary, NC, USA).

\section{Results}

\section{The respondents' characteristics}

Of the 1423 physicians contacted, 157 (11\%) replied. Physicians working in university hospitals accounted for $50 \%$ of the respondents, whereas $45 \%$ of the respondents worked in public-sector general hospitals and $5 \%$ worked in private for-profit or non-profit hospitals. They had a median of 10 years [418] of experience in the ICU. Most of the physicians had trained in critical care medicine (49\%) and anesthesiology (39\%).

\section{Crrt Practices}

Concerning renal replacement therapy (RRT), CRRT was preferred to intermittent hemodialysis (70\%). The CRRT techniques used by intensivists were variously venovenous hemofiltration (73\%), venovenous hemodiafiltration (57\%), venovenous hemodialysis (55\%), and Sustained Low-Efficiency Dialysis (8\%). Half of the physicians (52\%) reported prescribing continuous venovenous hemofiltration preferentially, followed by continuous venovenous hemodialysis (35\%). $64 \%$ of the respondents used citrate as the anticoagulant. $77 \%$ of the physicians prescribing hemofiltration reported using a combined predilution 
and postdilution modality. The median prescribed dose at initiation was $30 \mathrm{~mL} / \mathrm{kg} / \mathrm{h}$, and $75 \%$ of the prescribers considered the total body weight (with 39\% for body weight on admission and $36 \%$ for body weight on the day of the CRRT prescription). $12 \%$ of the respondents reported prescribing a flow effluent irrespective of body weight. Polyacrylonitrile was the most commonly used membrane material $(61 \%$, $40 \%$ of which were polyethylenimine-coated), followed by polysulfone (39\%).

\section{Beta-lactam Dosing Regimens During Crrt}

Concerning beta-lactam prescriptions during CRRT, the majority of the physicians (56\%) did not adjust the doses. $17 \%$ and $9 \%$ of them prescribed full doses for 24 and 48 hours, respectively, before reducing the dosing regimens. $13 \%$ of respondents reported prescribing a single loading dose before dose adjustment and $4 \%$ reported that their use of a reduced dose or a full dose depended on the antimicrobial agent in question. Only three physicians reported using reduced doses all the time. A dose adjustment could be either empirical or adapted according to the TDM results. In $85 \%$ of cases, the respondents did not adapt the beta-lactam dosing as a function of the CRRT dose or the effluent flow (85\%). The physicians used mainly the French renal prescription handbook (Guide Prescription et Rein) (74\%), TDM (62\%), and data from the literature (35\%) to adjust the beta-lactam dosing regimens. Only 4 physicians reported using pharmacokinetic software tools. When TDM was used, $100 \% \mathrm{~T}>4 \mathrm{MIC}$ was the most common pharmacokinetic/pharmacodynamic (PK/PD) target. $41 \%$ of the respondents sometimes consulted another specialist when deciding whether or not to adjust the beta-lactam dose during CRRT: this was variously an infectious disease specialist (29\%), a microbiologist (10\%), a pharmacist/pharmacologist (8\%), a nephrologist or toxicologist (5\%). The replies to the clinical vignettes highlighted a broad range of dose adaptation practices - particularly for meropenem (Fig. 1). Most participants used prolonged and continuous infusions (88\%), especially for piperacillin-tazobactam ( $91 \%)$, ceftazidime (71\%), cefotaxime (53\%), cefepime (50\%) and meropenem (40\%). Only $21 \%$ of physicians reported having access to a procedure for determining beta-lactam dosing regimens during CRRT. Only $34 \%$ of the physicians had the feeling that neurotoxicity was more frequent during CRRT. The use of TDM was significantly associated with prolonged and continuous infusions $(p=0.016)$ and a call to other specialists for advice on antibiotic dosing regimen adjustment during CRRT $(p<0.0001)$ (Table 2$)$. 
Table 2

Factors associated with the use of therapeutic drug monitoring

Use of therapeutic drug monitoring

$\begin{array}{lll}\text { Yes } & \text { No } & \text { p- } \\ n= & n= & \text { value }\end{array}$

(\%) (\%)

Type of institution

University hospital

$\begin{array}{lll}45 & 33 & 0,569\end{array}$

Other types of hospital

$41 \quad 38$

Years of experience $\leq 10$

Yes

$48 \quad 48$

0,493

No

$38 \quad 29$

No adjustment of dosing regimens during CRRT

Yes

48

45

0,425

No

$38 \quad 26$

Do you adjust the dosage according to the dialysis dose or effluent flow?

Yes

$13 \quad 10$

1,000

No

$72 \quad 59$

Do you call other specialists for advice on antibiotic dosing regimen adjustments for patients on CRRT?

Yes

$47 \quad 18<$

No

$18 \quad 53$

Does your institution have procedures for adjusting antibiotic doses in patients on CRRT?

Yes

$17 \quad 16 \quad 0,821$

No

$69 \quad 55$

Do you use prolonged/continuous infusions for beta-lactams in patients on CRRT?

Yes

$81 \quad 57 \quad 0,016$

The various groups were compared using Pearson's chi-squared test with Yates' correction. The threshold for statistical significance was set to $p<0.05$. 


\section{Use of therapeutic drug \\ monitoring}

No

5

14

The various groups were compared using Pearson's chi-squared test with Yates' correction. The threshold for statistical significance was set to $p<0.05$.

\section{Discussion}

To the best of our knowledge, the present study is the first to have assessed beta-lactam dosing practices in the context of CRRT. Furthermore, the survey described CRRT practices in France. Physicians practicing intensive care medicine (whatever their initial qualification) were included in the survey. The majority were intensivists or anesthesiologists, which reflects the fact that anesthesiologists are qualified for critical care medicine in France.

In order to review beta-lactam dosing in CRRT, we analyzed compliance with the French guidelines and Kidney Disease: Improving Global Outcomes (KDIGO) guidelines [21-22]. Our present findings were in line with studies of CRRT practices performed over the last decade [23-25]. CRRT was preferred to intermittent hemodialysis, although there is no clear evidence of superiority concerning reduced mortality [21-22]. This technique is considered to provide greater hemodynamic stability. As recommended, citrate was the main anticoagulant used [21-22]. Multicenter randomized controlled trials and meta-analyses have shown that increasing the CRRT dose intensity above $20-25 \mathrm{ml} / \mathrm{kg} / \mathrm{h}$ does not increase survival but does lead to more metabolic complications [26-29]. Therefore, in order to deliver a dose of 20-25 $\mathrm{ml} / \mathrm{kg} / \mathrm{h}$ and to minimize interruptions in the CRRT, the KDIGO guidelines recommend a value of $25-30$ $\mathrm{ml} / \mathrm{kg} / \mathrm{h}$ [22]. Although citrate limits filter coagulation, a third of our respondents indicated (as also found in other studies) that they prescribe a higher CRRT dose - leading to greater clearance of beta-lactams [23, 25]. The physicians reported using different reference body weights to prescribe the CRRT dose, which contributed to disparities in RRT practices. Although most studies are based on total weight at the time of randomization, the guidelines do not specify the weight to be used to determine the dialysis dose resulting in a variety of practices. Our survey highlighted the diversity and lack of harmonization of CRRT techniques.

Physicians may not be sufficiently aware of the need to maintain beta-lactam full doses during the initial phase of treatment. Administration must be optimized in order to maintain effective antibiotic concentrations for a sufficiently long time and thus maximize the chances of therapeutic success. This is especially true in the initial phase of sepsis when cardiac output and capillary permeability increase and protein binding is altered; this leads to increased clearance and a larger volume of distribution, inducing low serum and tissue beta-lactams concentrations $[1,30]$. Underexposure increases the likelihood of therapeutic failure and the emergence of resistance. Improving antibiotic exposure is, therefore, a major challenge. Secondly, organ dysfunctions (and especially kidney failure) lead to high serum antibiotic concentrations. CRRT may limit underdosing when using full doses with a potential risk of neurotoxicity 
in the event of overdosing $[4,12,15]$. The mechanisms by which antibiotics are eliminated by CRRT appear to be poorly understood since the majority of physicians do not reportedly adjust the dosage as a function of the dialysis dose or effluent flow.

Our survey highlighted the broad implementation of extended and continuous infusions and so showed that physicians were well aware of the time-dependent nature of the beta-lactams' activity. Even though the guidelines recommending the use of extended and continuous infusions for all compounds (to increase the probability of target attainment), these modalities are still mainly used for a few betalactams only and adherence to guidelines is suboptimal $[5,20]$. This might be due to the recent changes in the French guidelines between 2014 and 2018. The French-speaking Intensive Care Society initially recommended continuous infusions for ceftazidime and extended infusions (over 3-4 hours) for a few other beta-lactams [31]. In October 2018, the French Society of Anesthesia and Intensive Care Medicine and the French Society of Pharmacology and Therapeutics suggested the use of prolonged or continuous infusion of beta-lactams (intending to increase the probability of PK/PD target attainment and clinical cure rates) but did not differentiate between the various molecules [5]. These guidelines apply to infections with high-MIC bacteria or with non-fermenting Gram-negative bacilli, in patients in shock or with high severity scores, and lower respiratory tract infections (as described in our clinical vignettes). However, these guidelines were published just a few months before our survey, which may have limited their dissemination. Continuous and extended infusions are mostly used for piperacillin-tazobactam and ceftazidime; these are the compounds for which we have the most literature data, as reported in the ANTIBIOPERF study performed in 2015 [32]. Continuous infusion is mentioned in the French summary of product characteristics for ceftazidime only [33]. In the last decade, several other surveys have focused on these practices. A survey of 34 Belgian hospitals in 2011 showed that four beta-lactams were administered in the ICU by continuous and extended infusion to a varying extent: in $35 \%$ of the ICUs for cefepime, $38 \%$ for piperacillin-tazobactam, $68 \%$ for meropenem, and $81 \%$ for ceftazidime [34]. In 2013, an international multicenter survey of 402 physicians in 53 countries reported that piperacillin/tazobactam and carbapenem (meropenem and imipenem) were mainly administered as intermittent infusions (71\% and $68 \%$, respectively) [35]. In 2019, a German study reported that meropenem (70\%), piperacillin/tazobactam (67\%) and imipenem (50\%) were the beta-lactams most regularly administered as prolonged and continuous infusions [36]. A survey performed in Australia and New Zealand in 2016 focused on meropenem and piperacillin-tazobactam, which were most frequently administered in intermittent infusions [37]. The administration method may therefore differ from one geographic region to another. The survey of Australia and New Zealand highlighted the fact that the infusion modality could be determined on a case-by-case basis, depending on the presence of bacteria with high MICs, pathological changes (sepsis or sepsis shock), and the severity of the patient's illness [37].

The prescription tools used by the majority of physicians are not designed to recommend personalized dosages, and the use of pharmacokinetic software for finer dosage adjustment is rare. However, betalactam TDM is increasingly being used in CRRT, in line with the guidelines $[5,20]$. The 2018 French guidelines suggested performing TDM for ICU patients with expected variability in beta-lactam pharmacokinetics and/or in patients with clinical signs potentially related to beta-lactam toxicity [5]. The 
2020 European guidelines recommended TDM of beta-lactams as a standard of care in critically ill adult patients [20]. The fact that $56 \%$ of the physicians in our survey reported using full beta-lactam doses during CRRT and $62 \%$ mentioned using TDM suggests that beta-lactam TDM is often used only to determine the etiology of the neurotoxicity and that poor availability of TDM technique and the long wait for results from subcontractors limits the use of TDM in practice. This limitation has also been highlighted by other surveys [32, 35-36, 38]. TDM requires complex analytical systems (such as highperformance liquid chromatography or liquid chromatography/mass spectrometry) associated with high equipment and personnel costs. TDM is mostly performed for meropenem (22\%) and piperacillin (17\%) but is available for other beta-lactams in less than $5 \%$ of hospitals [36]. A lack of access to TDM at the weekend, the perception of a wide therapeutic range, and the relatively recent awareness of the clinical implications of beta-lactam underdosing were major hindrances to implementation [35-36]. Moreover, MICs for beta-lactams are not measured on a routine basis, which prevents antibiotic therapy from being adapted accordingly. Indeed, European Committee on Antimicrobial Susceptibility Testing (EUCAST) breakpoints are reported by laboratories in categories (i.e. susceptible, intermediate, or resistant) [36, 38]. An international survey of 328 hospitals in 53 countries performed in 2013 showed that TDM of piperacillin/tazobactam and meropenem was rare [35]. A French survey performed in 2015 found that beta-lactam TDM was available to $21 \%$ of the 507 respondents [32]. In 2019, a German survey showed that piperacillin, meropenem and ceftazidime were the beta-lactams most commonly dosed with TDM especially in patients undergoing RRT [38]. Overall, these surveys highlighted differences in beta-lactam TDM practices, patient selection, PK/PD targets, drug assay methods, and dose adjustment strategies.

The diversity of PK/PD targets emphasizes the uncertainties in the literature data, even though most physicians are well aware that a beta-lactam concentration over several times the MIC is required throughout the dosing interval (as already reported by Wong et al. in 2014 [38]). However, few of our respondents answered this question, showing that the concept of dosage adjustment based on PK/PD indices is poorly known (as already described in the ONTAl study [36]). In 2018, the French Society of Pharmacology and Therapeutics and the French Society of Anesthesia and Intensive Care Medicine suggested that targeting a free plasma beta-lactam concentration over four times the MIC of the causative bacteria (or the EUCAST epidemiological cut-off, when the MIC of the isolated strain is not available) for $100 \%$ of the dosing interval would maximize the bacteriological and clinical responses in critical care patients, whereas the European guidelines recommend a PK/PD index of between two and five times the MIC [5].

The duration of the beta-lactam infusion was not defined in the questionnaire's clinical vignettes; this limited the interpretation of our respondent's practices but highlighted the diversity of dosing regimens used. Although meropenem is the best-studied beta-lactam in CRRT, its dosing regimens differed most significantly among our respondents (Fig. 1, Additional file 1). This variability was also evidenced in a Belgian study of ICUs and non-ICU wards [34].

Moreover, our survey results underlined the need for procedures and the importance of a multidisciplinary approach for providing stable infusions, since most physicians are not aware of the stability data [32, 34]. 
The fact that respondents using TDM were more likely to call other specialists and more likely to use prolonged/continuous infusions shows that TDM is part of a comprehensive, complex approach to PK/PD optimization, which also requires determination of the MIC and knowledge of the PK/PD target.

The TDM included in Bayesian software represents the best option for personalizing antimicrobial dosing by taking account of various parameters (the MIC, site of infection, weight, renal function, severity, etc.). However, not all the clinical scenarios are available, and the high level of sophistication of these pharmacokinetic tools limits their implementation. Further studies are required to validate these tools and their potential clinical impact. Moreover, the variety of selected PK/PD targets raises the question of whether TDM is useful, given the resulting differences in dose adjustments. The different perceptions of neurotoxicity in patients on CRRT are probably related to the diversity of doses used, as illustrated in the replies to the clinical vignettes.

Our survey had some limitations. Firstly, the questionnaire did not fully reflect the complexity of having to decide on the dosing regimen at the bedside. The clinical vignettes and questions were simple and standardized. Secondly, the low response rate (11\%) was explained by recruitment bias, since the survey was conducted during the summer vacation. Thirdly, we did not have an exhaustive list of intensivists in France. However, all types of hospitals (university or not, public or private sector, etc.) were represented. Despite these limitations, we undertook the largest yet study of this type in French ICUs. The diversity of replies to our questionnaire highlights the uncertainties regarding dosage adjustments required in CRRT and the lack of harmonization of PK/PD targets and emphasized the need for further research on a topic that is crucial in critically ill patients.

\section{Conclusion}

The diversity of beta-lactam dosing regimens and the tools used to adjust it may be responsible for significant morbidity and mortality. Optimal antimicrobial dosing during CRRT remains challenging. Personalized TDM and the use of Bayesian softwares appear to be fundamental for optimizing betalactam dosing regimens and improving patients' outcomes. However, low availability and a lack of clinical validation limit the implementation of these tools.

\section{Abbreviations}

CRRT

continuous renal replacement therapy

EUCAST

European Committee on Antimicrobial Susceptibility Testing

ICU

intensive care unit

IQR

interquartile range

KDIGO

Page 15/20 
Kidney Disease:Improving Global Outcomes

MIC

minimal inhibitory concentration

PK/PD

pharmacokinetic/pharmacodynamic

RRT

renal replacement therapy

TDM

therapeutic drug monitoring

\section{Declarations}

\section{Ethics approval and consent to participate}

Not applicable.

\section{Consent for publication}

Not applicable.

\section{Availability of data and material}

The datasets used and/or analysed during the current study are available from the corresponding author on reasonable request.

Competing interests

\section{The authors declare that they have no competing interests.}

\section{Funding}

None.

\section{Authors' contributions}

Conceptualization, methodology: EM, JL and FL; formal analysis, investigation and writing - original draft preparation: EM; statistical analysis: GW; review and editing : JL, GW and FL; supervision : JL and FL.

\section{Acknowledgments}

We thank all the physicians who participated in the survey.

\section{References}


1. Roberts JA, Paul SK, Akova M, et al. DALI: defining antibiotic levels in intensive care unit patients: are current $\beta$-lactam antibiotic doses sufficient for critically ill patients? Clin Infect Dis. 2014;58(8):107283. doi:10.1093/cid/ciu027.

2. Taccone FS, Laterre P, Dugernier T, et al. Insufficient $\beta$-lactam concentrations in the early phase of severe sepsis and septic shock. Crit Care. 2010;14(4):R126. doi:10.1186/cc9091.

3. Delattre IK, Taccone FS, Jacobs F, et al. Optimizing $\beta$-lactams treatment in critically-ill patients using pharmacokinetics/pharmacodynamics targets: are first conventional doses effective? Expert Rev Anti Infect Ther. 2017;15(7):677-88. doi:10.1080/14787210.2017.1338139.

4. Hites $\mathrm{M}$, Taccone FS, Wolff $F$, et al. Case-control study of drug monitoring of $\beta$-lactams in obese critically ill patients. Antimicrob Agents Chemother. 2013;57(2):708-15. doi:10.1128/AAC.01083-12.

5. Guilhaumou R, Benaboud S, Bennis Y, et al. Optimization of the treatment with beta-lactam antibiotics in critically ill patients-guidelines from the French Society of Pharmacology and Therapeutics (Société Française de Pharmacologie et Thérapeutique-SFPT) and the French Society of Anaesthesia and Intensive Care Medicine (Société Française d'Anesthésie et Réanimation-SFAR). Crit Care Med. 2019;23(1):104. doi:10.1186/s13054-019-2378-9.

6. Sime FB, Roberts MS, Peake SL, et al. Does Beta-lactam Pharmacokinetic Variability in Critically III Patients Justify Therapeutic Drug Monitoring? A Systematic Review. Ann Intensive Care. 2012;2:35. doi:10.1186/2110-5820-2-35.

7. Shaw AR, Chaijamorn W, Mueller BA. We Underdose Antibiotics in Patients on CRRT. Semin Dial. 2016;29(4):278-80. doi:10.1111/sdi.12496.

8. Ruiz J, Favieres C, Broch MJ, et al. Individualised antimicrobial dosing in critically ill patients undergoing continuous renal replacement therapy: focus on total drug clearance. Eur J Hosp Pharm. 2018;25(3):123-6. doi:10.1136/ejhpharm-2016-001114.

9. Seyler L, Cotton F, Taccone FS, et al. Recommended $\beta$-lactam regimens are inadequate in septic patients treated with continuous renal replacement therapy. Crit Care. 2011;15(3):R137. doi:10.1186/cc10257.

10. Wong W, Choi G, Gomersall CD, et al. To increase or decrease dosage of antimicrobials in septic patients during continuous renal replacement therapy: the eternal doubt. Curr Opin Pharmacol. 2015;24:68-78. doi:10.1016/j.coph.2015.07.003.

11. De Waele JJ, Carlier M. Beta-lactam antibiotic dosing during continuous renal replacement therapy: how can we optimize therapy? Crit Care. 2014;18(3):158. doi:10.1186/cc13945.

12. Beumier M, Casu GS, Hites M, et al. $\beta$-lactam antibiotic concentrations during continuous renal replacement therapy. Crit Care. 2014;18(3):R105. doi:10.1186/cc13886.

13. Veiga RP, Paiva J. Pharmacokinetics-pharmacodynamics issues relevant for the clinical use of betalactam antibiotics in critically ill patients. Crit Care. 2018;22(1):233. doi:10.1186/s13054-018-2155-1.

14. Timsit J, Bassetti M, Cremer 0 , et al. Rationalizing antimicrobial therapy in the ICU: a narrative review. Intensive Care Med. 2019;45(2):172-89. doi:10.1007/s00134-019-05520-5. 
15. Roberts JA, Ulldemolins M, Roberts MS, et al. Therapeutic drug monitoring of beta-lactams in critically ill patients: proof of concept. Int J Antimicrob Agents. 2010;36(4):332-9. doi:10.1016/j.jjantimicag.2010.06.008.

16. Churchwell MD, Mueller BA. Drug dosing during continuous renal replacement therapy. Semin Dial. 2009;22(2):185-8. doi:10.1111/j.1525-139X.2008.00541.x.

17. Lewis SJ, Mueller BA. Antibiotic dosing in critically ill patients receiving CRRT: underdosing is overprevalent. Semin Dial. 2014;27(5):441-5. doi:10.1111/sdi.12203.

18. Li AMMY, Gomersall CD, Choi G, et al. A systematic review of antibiotic dosing regimens for septic patients receiving continuous renal replacement therapy: do current studies supply sufficient data? J Antimicrob Chemother. 2009;64(5):929-37. doi:10.1093/jac/dkp302.

19. Vaara S, Pettila V, Kaukonen K-M. Quality of pharmacokinetic studies in critically ill patients receiving continuous renal replacement therapy. Acta Anaesthesiol Scand. 2012;56(2):147-57. doi:10.1111/j.1399-6576.2011.02571.x.

20. Abdul-Aziz MH, Alffenaar JC, Bassetti M, et al, Infection Section of European Society of Intensive Care Medicine (ESICM); Pharmacokinetic/pharmacodynamic and Critically III Patient Study Groups of European Society of Clinical Microbiology and Infectious Diseases (ESCMID); Infectious Diseases Group of International Association of Therapeutic Drug Monitoring and Toxicology C (IATDMCT); Infections in the ICU and Sepsis Working Group of International Society of Antimicrobial Chemotherapy (ISAC). Antimicrobial therapeutic drug monitoring in critically ill adult patients: a Position Paper. Intensive Care Med. 2020;46(6):1127-1153. doi: 10.1007/s00134-020-06050-1.

21. Vinsonneau C, Allain-Launay E, Blayau C, et al. Renal replacement therapy in adult and pediatric intensive care: Recommendations by an expert panel from the French Intensive Care Society (SRLF) with the French Society of Anesthesia Intensive Care (SFAR) French Group for Pediatric Intensive Care Emergencies (GFRUP) the French Dialysis Society (SFD). Ann Intensive Care. 2015;5(1):58. doi:10.1186/s13613-015-0093-5.

22. Kidney Disease Improving Global Outcome KDIGO Acute kidney injury work group. KDIGO clinical practice guideline for acute kidney injury. Kidney Int Suppl. 2012;2:1-138.

23. Legrand $M$, Darmon $M$, Joannidis $M$, et al. Management of renal replaceent therapy in ICU patients: an international survey. Intensive Care Med. 2013;39(1):101-8. doi:10.1007/s00134-012-2706-x.

24. Guérin C, Girard R, Selli JM, et al. Intermittent versus continuous renal replacement therapy for acute renal failure in intensive care units: results from a multicenter prospective epidemiological survey. Intensive Care Med. 2002;28(10):1411-8. doi:10.1007/s00134-002-1433-0.

25. Digvijay K, Neri M, Fan W, et al. International Survey on the Management of Acute Kidney Injury and Continuous Renal Replacement Therapies: Year 2018. Blood Purif. 2019;47(1-3):113-9. doi:10.1159/000493724.

26. VA/NIH Acute Renal Failure Trial Network. Palevsky PM, Zhang JH, Connor TZ, et al.: intensity of renal support in critically ill patients with acute kidney injury. N Engl J Med. 2008;359(1):7-20. doi:10.1056/NEJMoa0802639. 
27. Bellomo R, Cass A, Cole L, et al. RENAL Replacement Therapy Study Investigators, Intensity of continuous renal-replacement therapy in critically ill patients. N Engl J Med. 2009;361(17):1627-38. doi:10.1056/NEJMoa0902413.

28. Joannes-Boyau O, Honore PM, Perez P, et al. High-volume versus standard-volume haemofiltration for septic shock patients with acute kidney injury (IVOIRE study): a multicentre randomized controlled trial. Intensive Care Med. 2013;39:1535-46. doi:10.1007/s00134-013-2967-z.

29. Van Wert R, Friedrich JO, Scales DC, et al. High-dose renal replacement therapy for acute kidney injury: systematic review and meta-analysis. Crit Care Med. 2010;38(5):1360-9. doi:10.1097/CCM.0b013e3181d9d912.

30. Ulldemolins M, Rello J. The relevance of drug volume of distribution in antibiotic dosing. Curr Pharm Biotechnol. 2011;12(12):1996-2001. doi:10.2174/138920111798808365.

31. Bédos $J$, Allaouchiche $B$, Armand-Lefèvre $L$, et al. Stratégies de réduction de l'utilisation des antibiotiques à visée curative en réanimation (adulte et pédiatrique) - Recommandations formalisées d'experts SRLF. Réanimation. 2014;23(5):558. /SFAR/GFRUP/SFM/SPILF/SF2H.

32. Charmillon A, Novy E, Agrinier N, et al. The ANTIBIOPERF study: a nationwide cross sectional survey about practices for $\beta$-lactam administration and therapeutic drug monitoring among critically ill patients in France. Clin Microbiol Infect. 2016;22(7):625-31. doi:10.1016/j.cmi.2016.04.019.

33. French public drug database. Summary of product characteristics FORTUM 2 g. Available at: http://base-donnees-publique.medicaments.gouv.fr/affichageDoc.php? specid=69443806\&typedoc=R. Accessed 26 February 2021.

34. Buyle FM, Decruyenaere J, De Waele J, et al. A survey of beta-lactam antibiotics and vancomycin dosing strategies in intensive care units and general wards in Belgian hospitals. Eur J Clin Microbiol Infect Dis. 2013;32(6):763-8. doi:10.1007/s10096-012-1803-7.

35. Tabah A, De Waele J, Lipman J, et al. Working Group for Antimicrobial Use in the ICU within the Infection Section of the European Society of Intensive Care Medicine (ESICM). The ADMIN-ICU survey: a survey on antimicrobial dosing and monitoring in ICUs. J Antimicrob Chemother. 2015;70(9):2671-7. doi:10.1093/jac/dkv165.

36. Liebchen U, Paal M, Scharf C, et al. The ONTAl study - a survey on antimicrobial dosing and the practice of therapeutic drug monitoring in German intensive care units. J Crit Care. 2020;60:260-6. doi:10.1016/j.jcrc.2020.08.027.

37. Cotta MO, Dulhunty JM, Roberts JA, et al. Should $\beta$-lactam antibiotics be administered by continuous infusion in critically ill patients? A survey of Australia and New Zealand intensive care unit doctors and pharmacists. Int J Antimicrob Agents. 2016;47(6):436-8. doi:10.1016/j.ijantimicag.2016.02.017.

38. Wong G, Brinkman A, Benefield RJ, et al. An international, multicentre survey of $\beta$-lactam antibiotic therapeutic drug monitoring practice in intensive care units. J Antimicrob Chemother. 2014;69(5):1416-23. doi:10.1093/jac/dkt523. 
Figures
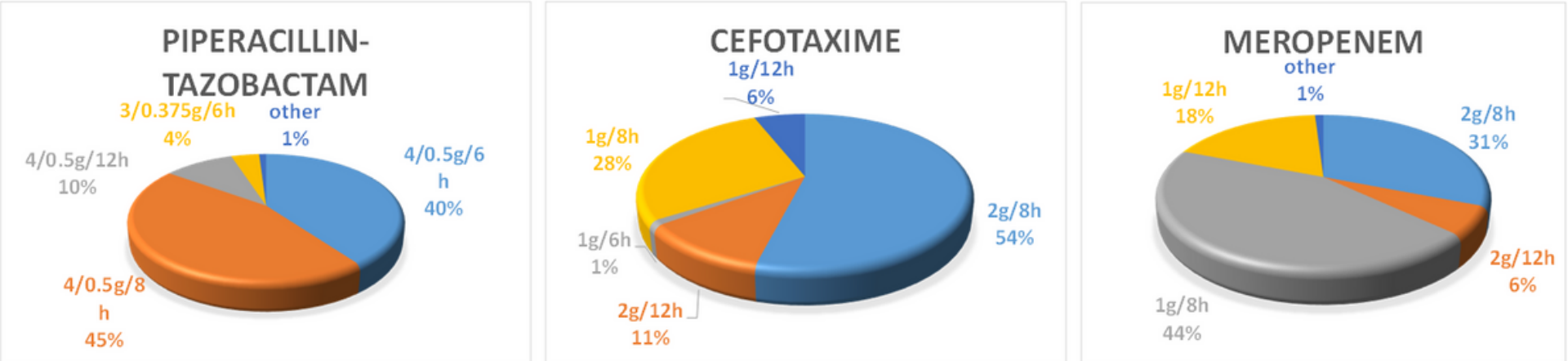

\section{Figure 1}

Summary of responses to the clinical vignettes in the study questionnaire

\section{Supplementary Files}

This is a list of supplementary files associated with this preprint. Click to download.

- supplementarymaterialcriticalcareVF.doc 